Rev Biomed 2002; 13:265-271.

\title{
Respuesta de novillas Brahman a la sincronización del estro con progestagenos; conducta sexual y tasa de gestación.
}

\section{Artículo Original}

Carlos Silva-Mena, Roberto Guzmán-Casas, Roger Delgado-León, Ricardo Aké-López.

Departamento de Reproducción y Mejoramiento Genético, Facultad de Medicina Veterinaria y Zootecnia, Universidad Autónoma de Yucatán, Mérida, Yucatán, México.

\section{RESUMEN.}

Introducción. La sincronización del estro es muy útil para implementar programas de inseminación artificial, facilitar el manejo de los animales y agruparlos para el servicio. La información acerca del uso de este procedimiento en razas cebuinas (Bos indicus) es relativamente escasa.

Objetivo. Evaluar el efecto del tratamiento con progestágenos sobre la presentación del estro, la conducta estral y la tasa de gestación en novillas Brahman servidas con monta natural.

Material y métodos. Se usaron 64 novillas distribuidas en cuatro grupos de 16 animales y cuatro toros de la misma raza. Se sincronizó el estro con Norgestomet y valerato de estradiol y se registró la actividad sexual observada durante 30 horas entre las novillas y entre ellas y el toro. La gestación se diagnosticó 50 a 60 días después.

Resultados. Entre el 81.2 y el $100 \%$ de las novillas presentaron estro en los cuatro grupos. A partir de las 40 horas de retirados los implantes, el estro tuvo una duración promedio ( \pm E.E.) de entre $8 \pm 1$ y $18 \pm$ 1.4 horas en los cuatro grupos. La tasa de preñez fue de 37.5 a $71.4 \%$ en los cuatro grupos.

Conclusión. Se puede sincronizar el estro hasta en un $90 \%$, en promedio, en novillas Brahman tratadas con Norgestomet + valerato de estradiol, con una duración e intensidad del estro variables y una tasa de gestación comparable con las observadas en hembras Bos taurus con tratamientos similares.

(Rev Biomed 2002; 13:265-271)

Palabras clave: Sincronización del estro, preñez, cebú, inseminación artificial.

\section{SUMMARY.}

Response of Brahman heifers to estrus synchronization with progestagens, sexual behavior and pregnancy rate.

Introduction. Estrus synchronization is a useful tool for artificial insemination programmes, for improving reproductive management and for grouping animals for service. Information on the procedure in Zebu cattle (Bos indicus) is relatively scarce.

Objective. To evaluate the effect of the treatment with progestagens on estrus presentation, estrus behavior and pregnancy rate in Brahman heifers with natural service.

Materials and methods. Sixty-four Brahman heifers, distributed in four groups $(\mathrm{n}=16)$ were used, as well

Solicitud de sobretiros: M. en C. Carlos Silva-Mena. Departamento de Reproducción Animal. Facultad de Medicina Veterinaria y Zootecnia, Universidad Autónoma de Yucatán. Km 15.5 carretera Mérida-Xmatkuil, A.P. 4-116 Itzimná, Mérida, Yucatán, México. Teléfono: 94232 13; Fax: 94232 05; Email: csilva@tunku.uady.mx

Recibido el 9/Julio/2001. Aceptado para publicación el 22/Noviembre/2001.

Este artículo está disponible en http://www.uady.mx/ biomedic/rb021345.pdf

Vol.13/No.4/Octubre-Diciembre, 2002 


\section{Silva-Mena, R Guzmán-Casas, R Delgado-León, R Aké-López.}

as four Brahman bulls. Estrus was synchronized with Norgestomet and estradiol valerate, and sexual activity observed during 30 hours among heifers and between heifers and the bull was recorded. Pregnancy was diagnosed 50 to 60 days later.

Results. Percentages of estrus presentation ranged from 81.2 to $100 \%$ in the four groups. Counted from 40 hours after implant removal, estrus length (mean \pm SEM) ranged from $8 \pm 1$ to $18 \pm 1.4$ hours. Rate of pregnancy of heifers serviced varied between 37.5 and $71.4 \%$.

Conclusion. Estrus can be synchronized up to an average of $90 \%$ in Brahman heifers treated with Norgestomet + estradiol valerate, with variable length and intensity and a resulting pregnancy rate with natural service comparable to that obtained in Bos taurus females treated similarly.

(Rev Biomed 2002; 13:265-271)

Key words: Estrus synchronization, pregnancy, Zebu.

\section{INTRODUCCIÓN.}

En los últimos años ha habido grandes avances en el conocimiento de la fisiología reproductiva del bovino, y en especial en el entendimiento del control hormonal de algunos eventos fisiológicos, como el que regula la presentación del estro y de la ovulación (1). El uso de hormonas específicas en circunstancias particulares contribuye a mejorar algunos aspectos de la reproducción; la administración de progestágenos sintéticos combinados con el valerato de estradiol es uno de estos procedimientos, el cual ha dado resultados satisfactorios para la sincronización del estro y la inducción del ciclo estral $(1,2)$.

La sincronización del estro no es un método para aumentar la fertilidad o la producción de crías sino que se usa como un instrumento de gran utilidad para implementar programas de inseminación artificial y/o facilitar el manejo de los animales y agruparlos para darles servicio (3). El método se puede usar también para programas de servicio con monta natural, con las ventajas de un mejor aprovechamiento de los toros y una concepción más temprana de las hembras.

Los resultados obtenidos con este método pueden variar de acuerdo con circunstancias como la raza y la condición corporal, entre otros. La mayor parte de la información sobre la utilización de este procedimiento se refiere a ganado Bos taurus en condiciones de climas templados; en los países de clima tropical su uso es más reducido, al igual que el número de estudios que se han realizado al respecto, por lo que la información sobre ese aspecto en el ganado Bos indicus, característico de las regiones tropicales, es considerablemente menor $(1,4)$.

El objetivo del presente estudio fue evaluar el efecto del tratamiento con progestágenos sobre la presentación del estro, la conducta estral y la tasa de gestación en novillas Brahman (Bos indicus) en condiciones de trópico.

\section{MATERIALES Y MÉTODOS.}

El estudio se hizo en un rancho del oriente del Estado de Yucatán, México. El clima de la región es cálido subhúmedo con temperatura promedio de $36^{\circ} \mathrm{C}$ y humedad relativa promedio del $80 \%$, con lluvias en verano y precipitación pluvial anual de entre $415 \mathrm{y}$ $1594 \mathrm{~mm}$ (5). Los grupos 1 y 2 se trataron en octubre de 1991 y de 1992, respectivamente, y los 3 y 4 en abril y en noviembre, respectivamente, de 1993.

Se usaron 64 novillas y cuatro toros Brahman (Bos indicus); las hembras nacieron en el mismo rancho en que se hizo el estudio, se destetaron a los ocho meses de edad y se alimentaron en pastoreo con zacate Guinea (Panicum máximum) y un suplemento de $2 \mathrm{~kg}$ de pollinaza por animal al día, humedecida con melaza diluida en agua. Se les desparasitó al destete y al año de edad. Además se les vacunó semestralmente contra derriengue, carbón sintomático y pasteurelosis. El hato estaba libre de brucelosis y de tuberculosis, lo que se verificaba mediante un control anual.

Las novillas se eligieron con base en un examen clínico para verificar el estado de salud y la normalidad del tracto reproductivo. Los animales de cada grupo de tratamiento eran contemporáneos y su edad era de alrededor de dos años y medio (29.9 \pm 0.82 meses); no se estableció si las novillas estaban o no ciclando y únicamente se efectuó un examen por

\section{Revista Biomédica}


Sincronización del estro con progestagenos.

palpación transrectal para verificar la normalidad del aparato reproductivo dos semanas antes del tratamiento. No se consideró el peso al momento del estudio, pero sí la condición corporal, que debía ser de 5 a 6 en una escala de 1 a 9, es decir de moderada a buena, de acuerdo con la metodología propuesta por Ayala y col (6).

Las 64 novillas se distribuyeron en cuatro grupos de 16 animales; este número se determinó con base en observaciones de otros autores acerca del número de vacas que sirve un toro en períodos de apareamiento cortos con estro sincronizado $(7,8)$. El estudio consistió en la sincronización del celo mediante un implante hormonal y la observación de la actividad sexual durante 30 horas a partir de las 40 horas de haber retirado el implante, en compañía de un toro de la misma raza previamente evaluado en cuanto a sus características reproductivas. El estro se sincronizó con un implante subcutáneo de Norgestomet en la cara externa de la oreja más una inyección intramuscular de $3 \mathrm{mg}$ de Norgestomet $+5 \mathrm{mg}$ de valerato de estradiol (Crestar, Intervet International Boxmeer, The Netherlands). A los 10 días se retiraron los implantes y se marcó a los animales con pintura brillante para facilitar su identificación. Cuarenta horas después se introdujo al toro en el grupo de novillas y se inició la observación de la conducta sexual.

La observación comenzó a las 0700 horas, en el corral de estancia de los animales y, para no alterar el manejo alimenticio de los mismos, continuó en un potrero de aproximadamente una hectárea a donde eran llevados a pastorear alrededor de las 1700 horas y de donde regresaban al corral hacia las 0600 horas del día siguiente. Se consideró que una novilla estaba en estro cuando se dejaba montar o montaba a otras novillas, y como final del estro el momento en que alguna de estas acciones ocurrió por última vez. La actividad sexual registrada incluyó las montas realizadas por las novillas, montas recibidas de otras novillas, montas recibidas del toro y los servicios recibidos. Las novillas servidas fueron palpadas 50 60 días más tarde para el diagnóstico de gestación. Para caracterizar más precisamente la respuesta a la sincronización, se analizó el porcentaje de animales en estro 40 horas después del retiro de los implantes, así como los porcentajes de animales en calor en cada periodo de 10 horas de las 30 horas de observación, además del porcentaje total de animales que presentaron calor. Se consideró como novilla en estro a las 40 horas después del retiro del implante a toda novilla vista en calor dentro de la primera hora de observación, o sea dentro de las 40 y las 41 horas después del retiro de los implantes.

Los datos se analizaron con el análisis de varianza de una vía complementada con la prueba de Duncan en los casos con diferencia significativa, el cálculo de coeficientes de correlación y la prueba de Chi Cuadrada, con la corrección de Yates en donde fue necesario $(9,10)$; también se calculó la media y el error estándar (E.E.) de los rasgos observados.

\section{RESULTADOS.}

Las características de la respuesta a la sincronización del estro se presentan en el cuadro 1 . El porcentaje de novillas en calor entre las 40 y las 70 horas después del retiro de los implantes varió entre el 81 y el $100 \%$, con una media de $90.6 \%$ y sin diferencias significativas $(\mathrm{p}>0.05)$ entre los grupos. A las 40 horas después del retiro de los implantes, entre el 43.7 y el $81.2 \%$ de las novillas ya estaban en

\section{Cuadro 1}

Porcentajes de novillas que presentaron estro y porcentajes de novillas en estro $\mathbf{4 0}$ horas después del retiro de los implantes y dentro de los tres siguientes lapsos de 10 horas.

\begin{tabular}{lllllll}
\hline \multicolumn{1}{c}{ Porcentaje } & $\mathbf{1}$ & $\mathbf{2}$ & $\mathbf{3}$ & $\mathbf{4}$ & $\begin{array}{c}\text { Promedio } \\
\mathbf{\%}\end{array}$ \\
\hline $\begin{array}{l}\text { Total de novillas } \\
\text { en calor }\end{array}$ & 81.2 & 87.5 & 93.7 & 90.6 \\
$\begin{array}{l}\text { Novillas en calor } 81.2 \\
\text { a las 40 horas }\end{array}$ & 68.7 & 43.7 & 56.2 & 62.4 \\
$\begin{array}{l}\text { Novillas en calor 100 } \\
\text { de 40 a 50 horas }\end{array}$ & 81.2 & 87.5 & 93.7 & 90.6 \\
$\begin{array}{l}\text { Novillas en calor 100a } \\
\text { de 50 a 60 horas }\end{array}$ & $56.2 \mathrm{~b}$ & $68.7 \mathrm{~b}$ & $6.2 \mathrm{c}$ & 57.8 \\
$\begin{array}{l}\text { Novillas en calo 81.2a } \\
\text { rde 60 a 70 horas }\end{array}$ & $0 \mathrm{~b}$ & $37.5 \mathrm{c}$ & $0 \mathrm{~b}$ & 25 \\
\hline
\end{tabular}

a,b,c, literales diferentes entre columnas indican diferencia significativa $(\mathrm{p}<0.05)$.

Vol.13/No.4/Octubre-Diciembre, 2002 


\section{Silva-Mena, R Guzmán-Casas, R Delgado-León, R Aké-López.}

estro en los cuatro grupos. Los mayores porcentajes de presentación del estro se observaron en el intervalo de 40 a 50 horas después del retiro de los implantes, o sea dentro de las primeras 10 horas de observación; del 81.2 al $100 \%$ de los animales presentaron calor en este lapso. En el segundo intervalo de 10 horas se observó un descenso del porcentaje de animales que presentaron calor, el cual varió entre el 6.2 y el $68.7 \%$, a excepción del grupo 1 que se mantuvo en el 100\%. En el último lapso de 10 horas de observación la actividad sexual fue nula en los grupos 2 y 4 y muy baja en el grupo 3, mientras que en el grupo 1 el porcentaje se mantenía todavía alto. Los porcentajes de novillas en estro difirieron significativamente entre los grupos $(\mathrm{p}<0.001)$ únicamente a las $50-60$ y las $60-70$ horas después del retiro de los implantes.

En el cuadro 2 se presentan las características del estro en los cuatro grupos de novillas, así como las de la interacción entre las novillas y el toro y la tasa de preñez. La duración del estro a partir de 40 horas después del retiro de los implantes varió entre 8 y 18 horas en términos generales, aunque en los grupos 2 al 4 la duración fue de entre 8 y 12 horas y el grupo 1 se apartó de los otros al tener una duración de 18 horas. Las diferencias fueron significativas entre el grupo 1 y los demás grupos ( $p<0.01)$, el 2 no difirió del 3 ni del 4 ( $p>0.05)$, pero los grupos 3 y 4 difirieron significativamente $(\mathrm{p}<0.05)$. El número de montas proporcionadas por cada novilla en estro varió entre 16 y 25 en los grupos 2 al 4, mientras que en el grupo 1 fue de 44, aunque en este caso sólo hubo diferencia significativa $(\mathrm{p}<0.01)$ entre el grupo 1 y los otros tres. Algo parecido ocurrió con el número de montas recibidas de otras hembras por cada novilla, el cual varió entre 13 y 26 en los grupos 2 a 4 y en el grupo 1 fue de cerca de 46, con diferencia significativa entre este último y los otros grupos ( $\mathrm{p}<0.01)$; además el grupo 4 difirió significativamente del $2(\mathrm{p}<0.05)$ y del 3 ( $\mathrm{p}<0.01)$. En cuanto al número de montas recibidas del toro por cada novilla en calor, éste varió entre prácticamente 2 y 10; el grupo 1 también se distinguió marcadamente de los otros por una mayor actividad, aunque las diferencias fueron significativas sólo entre los grupos 1 y 2 ( $\mathrm{p}<0.05)$. El número de servicios por novilla en estro fue similar en los cuatro grupos, ya que varió entre 0.9 y 1 , y el número de novillas que recibieron al menos un servicio fue de entre 7 y 12 , con el mayor número en el grupo 1, sin diferencia estadística $(p>0.05)$. Estos rasgos de la conducta sexual fueron muy variables dentro de cada grupo; el número de montas dadas por novilla tuvo un rango de 22 a 97 en el grupo 1; de 4 a 46 en el 2; de 0 a 44 en el 3 y de 2 a 33 en el 4 . El número de montas recibidas de otras novillas varió de 16 a 91; 1 a $80 ; 7$ a 24 y 0 a 37 en $\operatorname{los}$ grupos 1 a 4 , respectivamente. El número de montas y de servicios recibidos del toro por cada novilla fue de 0 a 36 y de 0 a $2 ; 0$ a 11 y 0 a $4 ; 0$ a 13 y 0 a 3 y 0 a 32 y 0 a 4 en los grupos 1 a 4, respectivamente. La tasa de preñez varió dentro de un rango relativamente amplio, ya que el menor porcentaje de gestación fue de 37.5 en el grupo 2 y el mayor fue de 71.4 en el grupo 4,

Cuadro 2

Duración (promedio \pm E.E.) del estro en los cuatro grupos de novillas Brahman, actividad sexual y tasa de preñez de las hembras servidas.

\begin{tabular}{|c|c|c|c|c|}
\hline Características & $1^{G}$ & $2^{\mathrm{U}}$ & $\mathbf{P}$ & $\mathrm{S}_{4}$ \\
\hline Duración del estro en horas & $18 \pm 1.4 \mathrm{a}$ & $10.1 \pm 0.5 b$ & $12.1 \pm 1.5 \mathrm{bc}$ & $8 \pm 1 \mathrm{bd}$ \\
\hline Montas dadas por novilla & $44.5 \pm 4.4 \mathrm{a}$ & $16.8 \pm 3.5 b$ & $24.8 \pm 4.1 \mathrm{~b}$ & $15.7 \pm 2.8 \mathrm{~b}$ \\
\hline No. de montas recibidas de otras novillas & $45.8 \pm 5.8 \mathrm{a}$ & $18.6 \pm 1.8 \mathrm{bc}$ & $26.7 \pm 6.2 \mathrm{bd}$ & $13.4 \pm 2.8 \mathrm{be}$ \\
\hline Montas recibidas del toro & $10.4 \pm 2.9 \mathrm{c}$ & $1.8 \pm 1 \mathrm{~d}$ & $4.2 \pm 1.06$ & $7.7 \pm 2.7$ \\
\hline Servicios por novilla & $0.9 \pm 0.2$ & $1.1 \pm 0.3$ & $1.07 \pm 0.3$ & $0.9 \pm 0.3$ \\
\hline No. (y \%) de novillas servidas. & $12(75)$ & $8(50)$ & $8(50)$ & $7(43.7)$ \\
\hline Tasa de preñez & $58.3 \%$ & $37.5 \%$ & $62.5 \%$ & $71.4 \%$ \\
\hline
\end{tabular}

Diferencia significativa entre columnas: a-b y e-d: $\mathrm{P}<0.01$; c-d $\mathrm{P}<0.05$ 
Sincronización del estro con progestagenos.

aunque esta diferencia no fue significativa $(\mathrm{p}>0.05)$.

En cuanto a la relación entre los rasgos de conducta sexual manifestados dentro de cada grupo en estudio, sólo se encontraron correlaciones significativas en tres de los grupos, pero éstas no fueron consistentes. En el grupo 4 el número de montas dadas por novilla se correlacionó con el número de montas recibidas de otras novillas $(\mathrm{r}=0.48, \mathrm{p}<0.10)$, y este último rasgo se correlacionó con el número de montas recibidas del toro por cada novilla $(\mathrm{r}=0.75$, $\mathrm{p}<0.01$ ). En los grupos 1 y 3 el número de montas dadas por el toro a cada novilla se correlacionó con el número de servicios recibidos por cada novilla $(\mathrm{r}=$ $0.59, \mathrm{p}<0.05$ y $\mathrm{r}=0.53, \mathrm{p}<0.10$, en los grupos 1 y 3 respectivamente).

\section{DISCUSION.}

El porcentaje de presentación de estro en este estudio está en los rangos reportados por otros autores con novillas Brahman en condiciones tropicales $(4,11,12)$. En climas templados y con animales tanto Bos indicus como Bos taurus se han obtenido resultados similares $(2,3,13,14)$. Esto sugiere que el uso de Norgestomet y valerato de estradiol puede dar buenos resultados en cualquiera de estas condiciones. Sin embargo, cabe señalar que los animales del presente trabajo tenían buena condición corporal y más de 2 años de edad. La condición corporal se debe cuidar siempre en los programas de sincronización de calores, ya que es un factor que puede influir sobre los resultados (13).

En cuanto a las proporciones de animales en estro en diferentes momentos después del retiro de los implantes, los presentes resultados concuerdan con los de Spitzer y col. (15) en ganado Bos taurus, ya que 48 horas después del retiro de los implantes estos autores observaron entre el 59 y el $88 \%$ de animales en calor; pero esta concordancia se pierde más tarde, ya que los mismos autores observaron que 72 horas después del retiro entre el 77 y el $90 \%$ de las vacas estaban en estro, mientras que en nuestro estudio el porcentaje ya era mucho más reducido de las 60 a 70 horas, e incluso en dos grupos ya no había signos de estro. La diferencia podría deberse a que en el Bos taurus el estro es más prolongado que en el Bos indicus, como han reportado algunos autores $(16,17)$. Por otra parte, el porcentaje de animales en estro a las 40 horas después del retiro del implante en el presente estudio es similar al observado por Corbet y col (14) entre las 25 y 48 horas después de retirar el implante en condiciones similares a las de este estudio, lo que sugiere que antes de las 40 horas el porcentaje de presentación de calores puede ser relativamente bajo.

Se ha reportado que la presencia del toro tiene un efecto estimulador sobre la presentación del estro por medio de mecanismos como la difusión de feromonas $(16,18)$. La introducción del toro 40 horas después del retiro de los implantes en el presente estudio pudo haber contribuido al aumento del porcentaje de manifestación de calores que se observó entre las 40 y las 50 horas.

La duración del estro observada en este estudio no se puede considerar como su duración total, ya que no se hizo la detección del estro antes de las 40 horas postretiro del implante y los datos indican que más del 50\% de los animales ya estaban en estro antes de ese momento. Aún así, la duración promedio del estro observada se asemeja a la reportada por otros autores, aunque los criterios usados por éstos para definir el inicio y el final del estro no hayan sido uniformes $(11,15,19)$. Según nuestros resultados y los de estos autores la duración del estro puede ser muy variable, probablemente debido a aspectos como el tipo de ganado y las condiciones en que se encuentra, entre otros, pero en general está comprendida entre las 4 y las 24 horas, con valores medios más frecuentes alrededor de las 10 a 18 horas.

Las diferencias observadas entre los grupos en cuanto a la duración del estro son difíciles de explicar, ya que los animales de los cuatro grupos eran similares en cuanto a edad, condición corporal y condiciones de manejo. Probablemente estas diferencias se deban a características particulares de los animales y a condiciones específicas de cada caso, lo que explicaría la amplia variación que se ha observado en general en cuanto a la duración del estro $(11,15,17)$.

La gran variación en la conducta sexual entre

Vol.13/No.4/Octubre-Diciembre, 2002 


\section{Silva-Mena, R Guzmán-Casas, R Delgado-León, R Aké-López.}

grupos y dentro de grupos sugiere que en cada caso se pueden observar diferencias marcadas en el comportamiento de los individuos, aunque el porcentaje total de animales que presentan estro parece ser bastante uniforme. Las medias de actividad sexual indican que cuando el estro dura más tiempo es mayor el número de montas que dan y reciben las novillas, lo cual parece lógico pero puede no ocurrir en todos los casos, ya que depende de la frecuencia con que se manifieste la actividad.

En general, cuanto más montas daba una hembra más montas recibía, pero en todos los grupos hubo hembras que mostraron una mayor tendencia a dar o a recibir montas; entre el 20 y el $79 \%$ de las novillas de cada grupo tuvieron diferencias mayores de 10 entre el número de montas dadas y el de recibidas, con algunos casos de novillas que tuvieron diferencias de entre 30 a 70 . El mostrar una mayor tendencia a montar o a ser montada durante el estro podría estar relacionado con el temperamento de las hembras, ya que se ha observado que algunas son dominantes o pasivas durante el estro, es decir que algunas tienden más a montar que a ser montadas cuando están en estro, mientras que otras tienden más a dejarse montar durante este período $(16,20)$.

Las correlaciones entre los rasgos de la conducta sexual refuerzan la observación de que no hay consistencia en la manifestación de los mismos dentro de los grupos, ya que no hubo uniformidad en las relaciones observadas. La correlación entre el número de montas recibidas del toro y el número de servicios recibidos, sugiere que mientras más activo sea un toro más servicios efectuará, aunque esto aparentemente sólo ocurrió en dos tratamientos. Esto se podría explicar por las diferencias entre los toros en cuanto a su eficiencia para efectuar el servicio (21).

El número de montas y de servicios del toro a cada novilla también fue muy variable. Estos rasgos dependen de la disposición de las hembras a dejarse montar y de la libido y capacidad de servicio del toro, así como de su eficiencia para efectuar el servicio, características que son muy variables y diferían significativamente entre los toros usados en este trabajo (21). La proporción de novillas servidas por grupo es semejante a la que se ha observado en estudios similares al presente $(7,22)$. En general, en períodos de apareamiento breves con estro sincronizado el toro no sirve a todas las vacas en calor, aunque el número de servicios que dé sea mayor que el de vacas en estro, lo cual se debe a que los toros tienden a servir varias veces a algunas hembras en lugar de distribuir sus servicios entre todas las hembras en calor; esta conducta parece estar relacionada con la capacidad de servicio de los toros y con la proporción vacas:toro (7, 22, 23).

En cuanto a la fertilidad, en el presente estudio hubo una variación relativamente amplia entre los grupos aunque el promedio, de alrededor del $57 \%$ de gestación, es similar al que observaron Koppel y Rodríguez (4) con inseminación artificial, y mayor que el observado por otros autores $(12,13)$ siempre con inseminación artificial. La tasa de preñez observada en el presente trabajo es equiparable a la que han observado algunos autores en ganado Bos taurus (8, $22,24,25)$, lo que sugiere que en algunas circunstancias la tasa de preñez del ganado Bos indicus puede asemejarse a las que se observan en ganado de razas europeas. En conclusión, los resultados del presente estudio indican que con Norgestomet y valerato de estradiol se puede sincronizar el estro en alrededor del $90 \%$ de los animales tratados, con una duración e intensidad variables de las manifestaciones de conducta sexual, y que la tasa de gestación con este tratamiento y servicio natural es comparable con lo que se ha observado incluso en ganado Bos taurus en condiciones similares.

\section{AGRADECIMIIENTOS.}

Los autores agradecen a la International Foundation for Science (IFS) su apoyo financiero para este estudio.

\section{REFERENCIAS.}

1. Porras AA, Galina H. Utilización de progestágenos para la manipulación del ciclo estral bovino. Vet Méx 1992; 23: 31-6.

2. González-Padilla E, Ruiz RL, Feber D, Denham A, Wiltbank JN. Puberty in beef heifers III Induction of fertile estrus. J Anim Sci 1975; 40:1110-8. 


\section{Sincronización del estro con progestagenos.}

3. Rodríguez ROL, González PE. Sincronización de 2 estros consecutivos e inseminación artificial sin detección del estro en vacas y vaquillas. Téc Pec Méx 1983; 44:52-7.

4. Koppel RET, Rodríguez ROL. Sincronización del celo con progestágenos e I.A. en vaquillas Cebú en el trópico. México Ganadero.1992; 359: 17-23

5. Gobierno del Estado de Yucatán. Monografía. Secretaría de Planeación. Mérida, Yucatán, México. 1983.

6. Ayala BA, Delgado LR, Honhold NY, Magaña MJ. A visual condition scoring scheme for Bos indicus and crossbred cattle. In: Dual Purpose Cattle Production Research", Eds, Anderson S, Wadsworth J. IFS (International Foundation for Science) / FMVZ-UADY 1995, Proceedings of an international workshop, March 1992, Mérida, México 1995; p. 119-28.

7. Williams GL. Breeding capacity, behavior and fertility of bulls with Brahman genetic influence during synchronized breeding of beef females. Theriogenology 1988; 30: 35-44.

8. Farin PW, Chenoweth PJ, Mateos ER, Pexton JE. Beef bulls mated to estrus synchronized heifers: single-vs multisire breeding groups. Theriogenology 1982; 17:365-71.

9. Snedecor GW, Cochran WG. Métodos estadísticos. Ed. Continental México. 1971.

10. Schwartz D. Méthodes Statistiques á L'usage des médecins et des biologistes. Flammarion Medecine Sciences. Paris. 1981.

11. Wishart DF, Young IM. Artificial insemination of progestin (SC1009) treated cattle at predeterminated times. Vet Rec 1974; 95: 503-8.

12. Salas-Vega E. Comportamiento reproductivo de novillas Brahman en respuesta al tratamiento con progestágenos en condiciones tropicales. Tesis de Maestría. Mérida, Yucatán, México. Universidad Autónoma de Yucatán. 1995

13. Menéndez TM, Robles BC, González PE. Sincronización del estro en vacas Cebú con o sin suplemento de melaza + urea. Tec Pec Méx 1977; 33: 9-14

14. Corbet NJ, Miller RG, Bindon BM, Burrow HM, Occhio MJ, Entwistle KW, et al. Synchronization of estrus and fertility in Zebu beef heifers treated with three estrus synchronization protocols. Theriogenology 1999; 51: 64759.
15. Spitzer JC, Burell WC, LeFever DG, Whitman RW, Wiltbank JN. Synchronization of estrus in beef cattle I. Utilization of Norgestomet implant and injection of Estradiol Valerate. Theriogenology 1978; 10: 181-00.

16. Galina CS, Orihuela A, Rubio I. Behavioural trends affecting oestrus detection in Zebu cattle. Anim Reprod Sci 1996; 42: 465-70.

17. Orihuela Trujillo JA. Conducta estral del ganado Cebú. Tesis de Maestría en Producción Animal, UNAM. 1982

18. Bolaños JM, Forsberg M, Kindahl H, Rodríguez-Martínez H. Biostimulatory effects of estrous cows and bulls on resumption of ovarian activity in postpartum anestrus Zebu (Bos indicus) cows in the humid tropics. Theriogenology 1998; 49: 629-38.

19. Pinheiro LO, Barros CM, Figueiredo RA, Do Valle ER, Encarnacao RO, Padovani CR. Estrous behavior and the estrus to ovulation interval in Nelore cattle (Bos indicus) with natural estrus induced with Prostaglandin F2alpha or Norgestomet and estradiol valerate. Theriogenology 1998; 49:667-81.

20. Peter AR, Ball PJM. Reproducción del ganado vacuno. Zaragoza: Acribia; 1991.

21. Silva-Mena C, Aké-López R, Delgado-León R. Sexual behavior and pregnancy rate of Bos indicus bulls. Theriogenology 2000; 53:991-1002.

22. Boyd GW, Lunstra DD, Corah LR. Serving capacity of crossbred yearling beef bulls. I single-sire mating behavior and fertility during average and heavy mating loads at pasture. J Anim Sci 1989; 67: 60-71.

23. Blockey Ma DeB. Serving capacity a measure of the serving efficiency of bulls during pasture mating. Theriogenology 1976; 6: 393-401.

24. Boyd GW, Healy VM, Mortimer RG, Piotrowski JR. Serving capacity tests are unable to predict the fertility of yearling bulls. Theriogenology 1991; 36: 1015-26.

25. Farin PW, Chenoweth PJ, Tomky DF, Pexton JE. Breeding soundness, libido and performance of beef bulls mated to estrus synchronized females. Theriogenology 1989; 32: $717-$ 25.

Vol.13/No.4/Octubre-Diciembre, 2002 